\title{
Is there a need and another way to measure the cosmic microwave background temperature more accurately?
}

\author{
J. Chluba ${ }^{1}$ and R. A. Sunyaev ${ }^{1,2}$ \\ 1 Max-Planck-Institut für Astrophysik, Karl-Schwarzschild-Str. 1, 85741 Garching bei München, Germany \\ e-mail: jchluba@mpa-garching.mpg.de \\ 2 Space Research Institute, Russian Academy of Sciences, Profsoyuznaya 84/32, 117997 Moscow, Russia
}

Received 2 July 2007 / Accepted 4 December 2007

\begin{abstract}
The recombination history of the Universe depends exponentially on the temperature, $T_{0}$, of the cosmic microwave background. Therefore tiny changes of $T_{0}$ are expected to lead to significant changes in the free electron fraction. Here we show that even the current $1 \sigma$-uncertainty in the value of $T_{0}$ results in more than half a percent ambiguity in the ionization history, and more than $0.1 \%$ uncertainty in the $T T$ and $E E$ power spectra at small angular scales. We discuss how the value of $T_{0}$ affects the highly redshifted cosmological hydrogen recombination spectrum and demonstrate that $T_{0}$ could, in principle, be measured by looking at the low frequency distortions of the cosmic microwave background spectrum. For this no absolute measurements are necessary, but sensitivities on the level of $\sim 30 \mathrm{nK}$ are required to extract the quasi-periodic frequency-dependent signal with typical $\Delta v / v \sim 0.1$ coming from cosmological recombination. We also briefly mention the possibility of obtaining additional information on the specific entropy of the Universe, and other cosmological parameters.
\end{abstract}

Key words. cosmology: cosmic microwave background - cosmology: theory - cosmology: cosmological parameters

\section{Introduction}

The recombination history of the Universe (Zeldovich et al. 1968; Peebles 1968; Seager et al. 1999, 2000) depends exponentially on the exact value of $T_{0}$ (Sunyaev \& Zeldovich 1970). Therefore one expects that tiny changes of $T_{0}$ lead to significant modifications of the ionization history. The temperature of the cosmic microwave background (CMB) was measured with tremendously high accuracy using the COBE/FIRAS instrument: $T_{0}=2.725 \pm 0.001 \mathrm{~K}$ (Fixsen \& Mather 2002) or $\Delta T / T \sim 0.04 \%$, where $1 \mathrm{mK}$ is the $1 \sigma$-error, or $\Delta T= \pm 2 \mathrm{mK}$ at $95 \%$ confidence (Mather et al. 1999). This value is based on the results obtained with the COBE/FIRAS and the quoted uncertainties from other methods, e.g. by measuring the CMB dipole anisotropy with the COBE/DMR instrument: $T_{0}=2.725 \pm$ $0.020 \mathrm{~K}$ (Kogut et al. 1996) or its slightly improved value $T_{0}=$ $2.725 \pm 0.012 \mathrm{~K}$ (Mather et al. 1999), are much larger, yielding $\Delta T / T \sim 0.7 \%$ and $\Delta T / T \sim 0.4 \%$ respectively. Also within the COBE/FIRAS instrument a systematic difference of $\Delta T \sim 5 \mathrm{mK}$ arose due to readout current heating (Mather et al. 1999), which was only understood later (Fixsen et al. 1996; Mather et al. 1999; Fixsen \& Mather 2002). The main goal of the present paper is to demonstrate that even changes of the order of a few $\mathrm{mK}$ lead to percent-level corrections in the ionization history, and that in principle there may be another indirect, but potentially accurate way to measure the CMB monopole temperature using the cosmological recombination spectrum coming from redshifts $z \gtrsim 800$

The impact of the uncertainty in the CMB monopole temperature on the CMB angular power spectra was addressed well before the era of precision cosmology ( $\mathrm{Hu}$ et al. 1995) with the conclusion that the corresponding theoretical error is below $\sim 1 \%$. However, the great success in observations of the CMB temperature and polarization anisotropies (Hinshaw et al. 2006; Page et al. 2006) renders this level of uncertainty insufficient for the analysis and interpretation of future CMB data, which will become available after the launch of the PLANCK Surveyor ${ }^{1}$, or with CMBPol. As explained in Seljak et al. (2003), the ultimate goal is to achieve $\sim 0.1 \%$ accuracy for the theoretical prediction of the CMB power spectra. In particular when discussing the imprints of different inflationary models on the power spectra, or when obtaining estimates of the key cosmological parameters that reach sub-percent accuracy, this precision becomes necessary.

Currently, in this context the largest uncertainty is considered to be due to our understanding of the epoch of cosmological recombination (e.g. Seljak et al. 2003). This fact has recently motived several studies on high precision computations of the cosmological hydrogen (Dubrovich \& Grachev 2005; Chluba \& Sunyaev 2006b; Kholupenko \& Ivanchik 2006; Rubiño-Martín et al. 2006; Chluba et al. 2007; Chluba \& Sunyaev 2007a,b), and helium (Wong \& Scott 2007; Switzer \& Hirata 2007a,b; Hirata \& Switzer 2007; Kholupenko et al. 2007; Rubino-Martin et al. 2007) recombination history. All the discussed additional physical processes lead to $\gtrsim 0.1 \%$ level corrections of the ionization history, which also partially cancel each other. The overall theoretical uncertainty in the CMB temperature and polarization power spectra, in particular at large $l$, still exceeds the level of $0.1 \%$. Here we show that the current $1 \sigma$-error in the value of $T_{0}$ also yields more than $0.1 \%$ uncertainty in the $T T$ and $E E$ power spectra.

\footnotetext{
1 www.rssd.esa.int/Planck
} 




Fig. 1. Ambiguity in the ionization history due to uncertainty in the value of $T_{0}$. We changed $T_{0}=2.725 \mathrm{~K}$ by $\Delta T$ as labeled. All the other parameters were not altered.

During the epoch of cosmological hydrogen recombination, roughly 5 photons are emitted per recombined hydrogen atom (Chluba \& Sunyaev 2006a). As realized earlier (e.g. see Zeldovich et al. 1968; Peebles 1968; Dubrovich 1975; Bernshtein et al. 1977; Dubrovich \& Stolyarov 1995; Burgin 2003; Dubrovich \& Grachev 2004; Kholupenko et al. 2005), the amount of these photons and their spectral distribution also depends on the parameters of the Universe. Here we demonstrate that for the cosmological recombination spectrum the dependence on the CMB monopole temperature, $T_{0}$, the total amount of baryons, $\propto \Omega_{\mathrm{b}} h^{2}$, and the abundance ratio of helium to hydrogen, $Y_{\mathrm{p}}$, are likely to be most important. Basing our analysis on the code developed in Rubiño-Martín et al. (2006) and Chluba et al. (2007), we illustrate that in principle, by measuring the cosmological recombination spectrum in the decimeter spectral band, one should be able to determine the value of $T_{0}$. Most importantly, for this no absolute measurements are necessary, but sensitivities on the level of $30 \mathrm{nK}$ are required to extract the quasi-periodic signal with typical $\Delta v / v \sim 0.1$ coming from cosmological recombination. For observations of the CMB angular fluctuations, a sensitivity level of $10 \mathrm{nK}$ in principle can be achieved (Readhead 2007). However, here the same signal is coming from every direction on the sky. Therefore one may use wide-angle horns, so that one is dealing with a huge flux of photons, carefully selecting regions on the sky that are cleanest with respect to foreground signals.

\section{Effect on the free electron fraction and $\mathrm{CMB}$ power spectra}

To study how the uncertainty in the value of $T_{0}$ affects the ionization history and CMB temperature and polarization power spectra, we used the CMBEASY code (Doran 2005). We ran our computations for a flat $\Lambda \mathrm{CDM}$ cosmological concordance model (Bennett et al. 2003) with $T_{0}=2.725 \mathrm{~K}, \Omega_{\mathrm{cdm}}=0.2234$, $\Omega_{\mathrm{b}}=0.0444, h=0.71$ and $Y_{\mathrm{p}}=0.24$.

In Fig. 1 we show the uncertainty in the free electron fraction, $N_{\mathrm{e}}$. As expected, a slightly higher value of $T_{0}$ delays recombination, while a smaller value shifts the time of recombination towards higher redshifts. Even for $\Delta T=1 \mathrm{mK}$, the change


Fig. 2. Ambiguity in the temperature (top panel) and $E$-mode polarization (lower panel) power spectra due to uncertainty in the value of $T_{0}$. We changed $T_{0}=2.725 \mathrm{~K}$ by $\Delta T$ as labeled. All the other parameters were not modified.

in the ionization history exceeds half a percent at $z \sim 950$. This shows that due to the exponential dependence of $N_{\mathrm{e}}$ on $T_{0}$ (Sunyaev \& Zeldovich 1970) a tiny error of $\Delta T / T_{0} \sim 0.04 \%$ is amplified by more than one order of magnitude. The maximal change in the free electron fraction roughly scales as $\Delta N_{\mathrm{e}} / N_{\mathrm{e}} \approx$ $15 \Delta T / T_{0} \approx 0.55 \% \times\left[\frac{\Delta T}{1 \mathrm{mK}}\right]$ for $\Delta T / T_{0} \ll 1$.

In Fig. 2 we show the corresponding uncertainty in the $T T$ and $E E$ power spectra. At large scales, the positions of the first peaks are slightly shifted, while at small scales $(l \gtrsim 1000)$, the actual modifications due to photon diffusion (Silk 1968) dominate. For earlier recombination $(\Delta T<0)$, more power is left in the $T T$ power-spectrum at small scales. On the other hand the amplitude of the $E$-mode polarization is lower, likely due to less scattering off moving electrons. In both cases the uncertainty is several times smaller than for the ionization history: even for $\Delta T= \pm 4 \mathrm{mK}$, where $\Delta N_{\mathrm{e}} / N_{\mathrm{e}}$ exceeds $2 \%$ close to its maximum, the power spectra are affected by less than a percent at $l \leq 3000$. For the $T T$ power spectrum one has $\Delta C_{l} / C_{l} \approx 2.1 \Delta T / T_{0}$ at $l \sim 450$ and $\Delta C_{l} / C_{l} \approx-3.5 \Delta T / T_{0}$ at $l \sim 3000$. Similarly for the $E E$ power spectrum one finds $\Delta C_{l} / C_{l} \approx-4.8 \Delta T / T_{0}$ at $l \sim 240$ and $\Delta C_{l} / C_{l} \approx 4.1 \Delta T / T_{0}$ at $l \sim 3000$.

The relative change is growing towards larger $l$, but at $l \gtrsim 1000-2000$ the signals connected with clusters of galaxies (Sunyaev \& Zeldovich 1970, 1972; and e.g. Springel et al. 2001; 


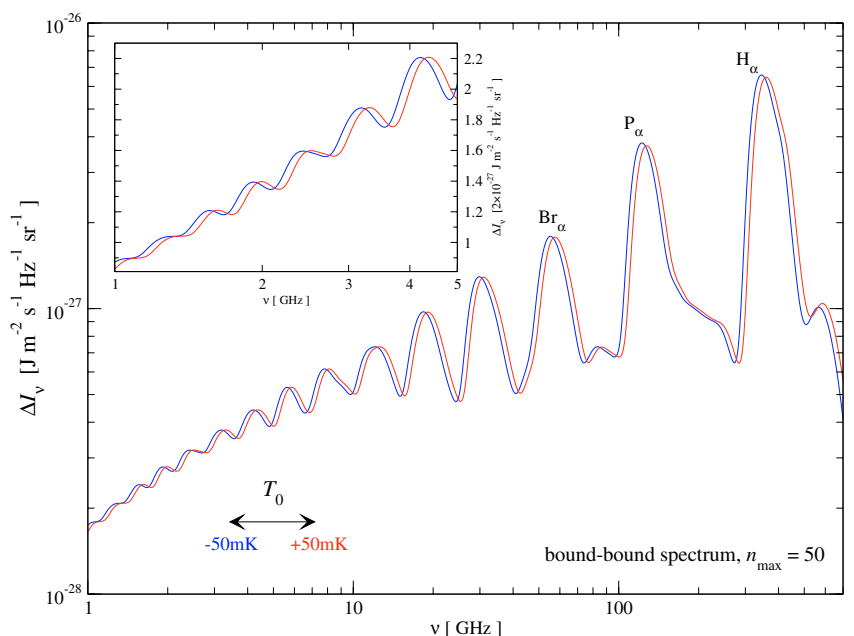

Fig. 3. Dependence of the bound-bound recombination spectrum on the value of $T_{0}$. We chose a large value for $\Delta T$ in order to illustrate the effect. All the other cosmological parameters remained unchanged. The results are based on computations including 50 shells for the hydrogen atom (see Rubiño-Martín et al. 2006 and Chluba et al. 2007, for computational details). The whole recombination spectrum is shifted along the frequency axis, but the overall normalization is not affected.

Schäfer et al. 2006), weak lensing, foregrounds, and (especially at high frequencies) ScUBA sources (Haiman \& Knox 2000) and starforming, merging, dusty galaxies (Righi et al. 2007) will start to be more important. Still, at $l \lesssim 1000-2000$ the multi- $l$ comparison of the observed CMB power spectra with the predicted theoretical mean model over a wide range of angular scales makes the discussed changes potentially important once systematic and statistical errors decrease with the improvements achieved using future CMB experiments. Furthermore, the effect under discussion has a similar order of magnitude as the corrections due to previously neglected physical processes (see Introduction), so it should be included in the list of mechanisms that lead to small modifications in the ionization history and CMB power spectra.

\section{Dependence of the recombination spectrum on different cosmological parameters}

In this section we illustrate the impact of different cosmological parameters on the hydrogen recombination spectrum. We restrict ourselves to the bound-bound emission spectrum and included 50 shells for the hydrogen atom into our computations. A more rigorous investigation is in preparation, however, the principle conclusions should not be affected.

In Fig. 3 we illustrate the dependence of the hydrogen recombination spectrum on the value of $T_{0}$. The value of $T_{0}$ mainly defines the time of recombination, and consequently when most of the emission in each transition appears. This leads to a dependence of the line positions on $T_{0}$, but the total intensity in each transition (especially at frequencies $v \lesssim 30 \mathrm{GHz}$ ) remains practically the same. The fractional shift of the low frequency spectral features along the frequency axis scales roughly as $\Delta v / v \sim \Delta T / T_{0}$. Hence $\Delta T \sim 1 \mathrm{mK}$ implies $\Delta v / v \sim 0.04 \%$ or $\Delta v \sim 1 \mathrm{MHz}$ at $2 \mathrm{GHz}$. Since the maxima and minima of the line features due to the large duration of recombination are rather broad $(\sim 10-20 \%)$, it is probably better to look for these shifts close to the steep parts of the lines, where the derivatives of the spectral distortion due to hydrogen recombination are largest. It
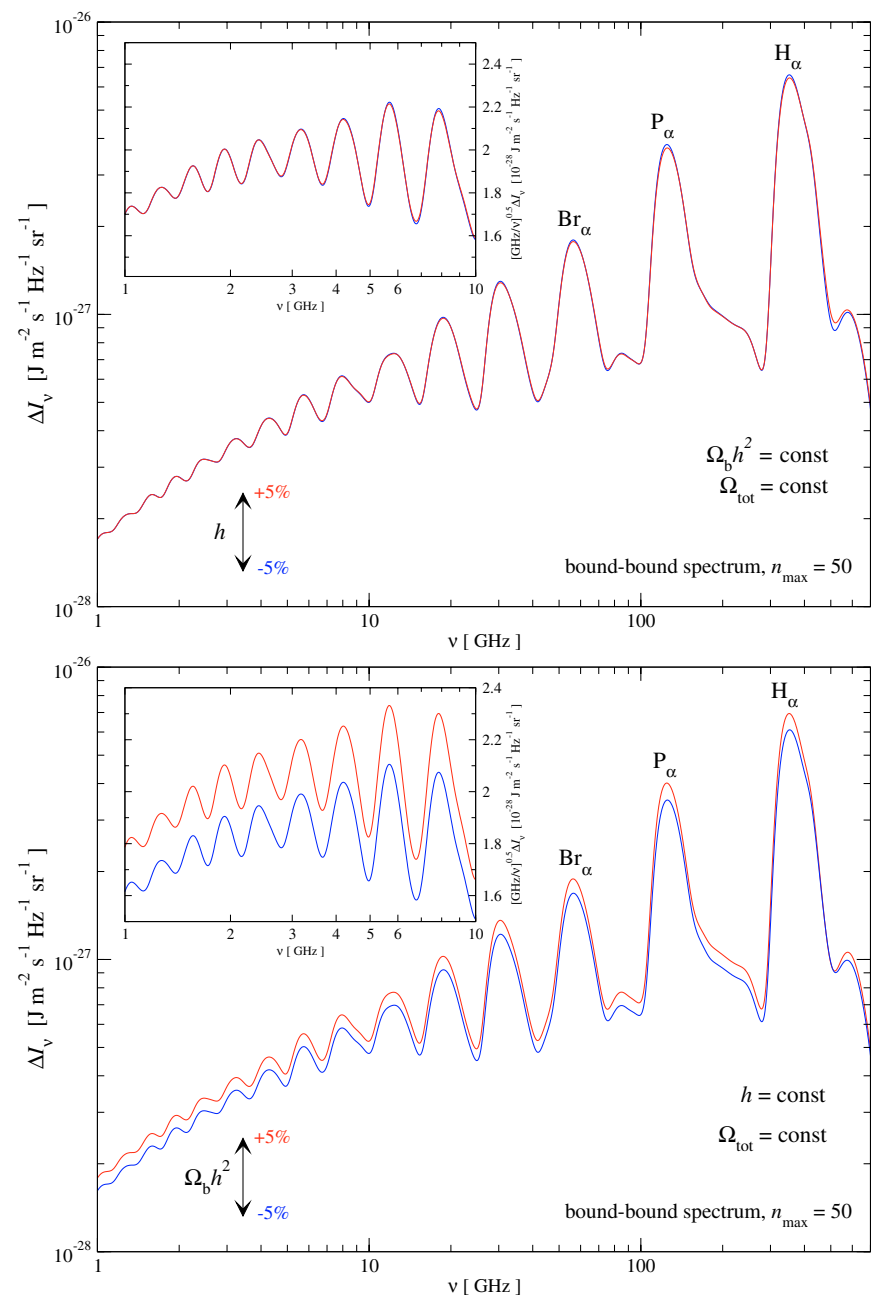

Fig. 4. Dependence of the bound-bound recombination spectrum on the number of hydrogen nuclei, $N_{\mathrm{p}} \propto \Omega_{\mathrm{b}} h^{2}$. In the upper panel we varied $h$, but fixed $\Omega_{\mathrm{b}} h^{2}$ and $\Omega_{\mathrm{tot}}=1$, while in the lower panel we changed $\Omega_{\mathrm{b}}$ keeping $h$ and $\Omega_{\mathrm{tot}}=1$ fixed. All results are based on computations including 50 shells for the hydrogen atom (see Rubiño-Martín et al. 2006 and Chluba et al. 2007, for computational details).

is also important to mention that the hydrogen recombination spectrum is shifted as a whole, allowing to increase the significance of a measurement by considering many spectral features at several frequencies.

The upper panel of Fig. 4 shows that at low frequencies the cosmological hydrogen recombination spectrum is practically independent of the value of $h$. Only the features due to the Lyman, Balmer, Paschen and Brackett series are slightly modified. This is connected to the fact that $h$ affects the ratio of the atomic time scales to the expansion time. Therefore changing $h$ affects the escape rate of photons in the Lyman- $\alpha$ transition and the relative importance of the $2 \mathrm{~s}-1 \mathrm{~s}$ transition. For transitions among highly excited states it is not crucial via which channel the electrons finally reach the ground state of hydrogen and hence the modifications of the recombination spectrum at low frequencies due to changes of $h$ are small. Changes of $\Omega_{\mathrm{m}} h^{2}$ should affect the recombination spectrum for the same reason.

The lower panel in Fig. 4 illustrates the dependence of the hydrogen recombination spectrum on $\Omega_{\mathrm{b}} h^{2}$. The total number of photons released during hydrogen recombination is directly related to the total number of hydrogen nuclei (e.g. Chluba \& Sunyaev 2006a). Therefore one expects that the overall 
normalization of the recombination spectrum depends on the total number of baryons, $N_{\mathrm{b}} \propto \Omega_{\mathrm{b}} h^{2}$, and the helium to hydrogen abundance ratio, $Y_{\mathrm{p}}$. Varying $\Omega_{\mathrm{b}} h^{2}$ indeed leads to a change in the overall amplitude $\propto \Delta\left(\Omega_{\mathrm{b}} h^{2}\right) /\left(\Omega_{\mathrm{b}} h^{2}\right)$. Similarly, changes of $Y_{\mathrm{p}}$ would affect the normalization of the hydrogen recombination spectrum, however it is important to also take the helium recombination spectrum into account. Changing $Y_{\mathrm{p}}$ will affect the relative contribution of the helium to the hydrogen recombination spectrum. Since the physics of helium recombination is different than in the case of hydrogen (e.g. the spectrum of neutral helium is more complicated; helium recombination occurs at earlier times, when the medium was hotter; He III $\rightarrow \mathrm{He}$ II is more rapid, so the recombination lines are narrower ${ }^{2}$ ), one can expect to find direct evidence of the presence of helium in the full recombination spectrum. These might be used to quantify the total amount of helium during the epoch of recombination, well before the first appearance of stars.

\section{Discussion and conclusions}

In Sect. 2 we have shown that the $1 \sigma$-error in the exact value of $T_{0}$ leads to more than half a percent ambiguity in the recombination history, resulting in more than $0.1 \%$ uncertainty in the $T T$ and $E E$ power spectra. In the analysis of future $C M B$ data one can easily include this additional source of uncertainty by considering the value of $T_{0}$ as a well-constrained free parameter.

It is clear that at the $\gtrsim 0.1 \%$ level, the uncertainties in the helium abundance ratio, $Y_{\mathrm{p}}$, and the effective number of neutrinos, $N_{v}$, should also be considered (e.g. Hu et al. 1995; Olive \& Steigman 1995; Cyburt 2004; Steigman 2006). In particular the value of $Y_{\mathrm{p}}$ has a strong impact, directly affecting the peak of the Thomson visibility function (Sunyaev \& Zeldovich 1970) due to the dependence of the number of hydrogen nuclei, $N_{\mathrm{H}}$, for a fixed number of baryons, on the helium abundance ratio $\left(N_{\mathrm{H}} \propto\left[1-Y_{\mathrm{p}}\right]\right)$. It is important to mention that the effects connected with the angular fluctuations of the CMB temperature $\left(\Delta T / T \sim\right.$ few $\left.\times 10^{-5}\right)$, as observed with COBE and WMAP, is expected to be one order of magnitude smaller.

In Sect. 3 we discussed the dependence of the cosmological hydrogen recombination spectrum on different cosmological parameters. We have illustrated that changes in the value of the CMB temperature, $T_{0}$, lead to an overall shift of the hydrogen recombination spectrum along the frequency axis, leaving the total normalization practically unchanged. On the other hand changes of the total amount of baryons, $\propto \Omega_{\mathrm{b}} h^{2}$, mainly affects the amplitude of the recombination signal. This in principle should allow measurement of both parameters separately. However, a more detailed analysis is required, also including the lines from cosmological helium recombinations (Rubino-Martin et al. 2007), and the free-bound and two-photon contributions. In this way it may be possible to construct an accurate spectral template, which can be used to disentangle the cosmological recombination spectrum from other astrophysical sources.

In addition to the interesting potential of constraining cosmological parameters (without suffering from limitations set by cosmic variance), an observation of the radiation from the epoch of recombination will yield the final proof that our theoretical understanding of how the Universe became neutral is correct. This aspect is particularly important when considering the

\footnotetext{
${ }^{2}$ Our computations show that the broadening due to electron scattering is $\Delta v / v \lesssim 7 \%$, so the overall width of the He III $\rightarrow \mathrm{He}$ IIfeatures is still smaller than the cosmological hydrogen recombination lines (Rubino-Martin et al. 2007).
}

possibility of non-standard recombination scenarios (Peebles et al. 2000; Doroshkevich et al. 2003; Bean et al. 2003, 2007), which would also affect the results obtained here.

Acknowledgements. The authors wish to thank E. E. Kholupenko for his comments on the paper, and J. R. Bond, C. Hernández-Monteagudo, J. A. Rubiño-Martín and B. D. Wandelt for useful discussions. We are grateful for discussions on experimental possibilities with J. E. Carlstrom, D. J. Fixsen, A. Kogut, M. Pospieszalski, A. Readhead, E. J. Wollack and especially J. C. Mather.

\section{References}

Bean, R., Melchiorri, A., \& Silk, J. 2003, Phys. Rev. D, 68, 083501 Bean, R., Melchiorri, A., \& Silk, J. 2007, Phys. Rev. D, 75, 063505 Bennett, C. L., Halpern, M., Hinshaw, G., et al. 2003, ApJS, 148, 1 Bernshtein, I. N., Bernshtein, D. N., \& Dubrovich, V. K. 1977, SvA, 21, 409 Burgin, M. S. 2003, Astron. Rep., 47, 709

Chluba, J., \& Sunyaev, R. A. 2006a, A\&A, 458, L29

Chluba, J., \& Sunyaev, R. A. 2006b, A\&A, 446, 39

Chluba, J., \& Sunyaev, R. A. 2007a, A\&A, 475, 109

Chluba, J., \& Sunyaev, R. A. 2007b, ArXiv e-prints, 705

Chluba, J., Rubiño-Martín, J. A., \& Sunyaev, R. A. 2007, MNRAS, 374, 1310

Cyburt, R. H. 2004, Phys. Rev. D, 70, 023505

Doran, M. 2005, J. Cosmol. Astro-Part. Phys., 10, 11

Doroshkevich, A. G., Naselsky, I. P., Naselsky, P. D., \& Novikov, I. D. 2003, ApJ, 586, 709

Dubrovich, V. K. 1975, SvA, 1, 196

Dubrovich, V. K., \& Grachev, S. I. 2004, Astron. Lett., 30, 657

Dubrovich, V. K., \& Grachev, S. I. 2005, Astron. Lett., 31, 359

Dubrovich, V. K., \& Stolyarov, V. A. 1995, A\&A, 302, 635

Fixsen, D. J., \& Mather, J. C. 2002, ApJ, 581, 817

Fixsen, D. J., Cheng, E. S., Gales, J. M., et al. 1996, ApJ, 473, 576

Haiman, Z., \& Knox, L. 2000, ApJ, 530, 124

Hinshaw, G., Nolta, M. R., Bennett, C. L., et al. 2006, ArXiv Astrophysics e-prints

Hirata, C. M., \& Switzer, E. R. 2007, ArXiv Astrophysics e-prints

Hu, W., Scott, D., Sugiyama, N., \& White, M. 1995, Phys. Rev. D, 52, 5498

Kholupenko, E. E., \& Ivanchik, A. V. 2006, Astron. Lett., 32, 795

Kholupenko, E. E., Ivanchik, A. V., \& Varshalovich, D. A. 2005, Gravitation Cosmol., 11, 161

Kholupenko, E. E., Ivanchik, A. V., \& Varshalovich, D. A. 2007, MNRAS, 378, L39

Kogut, A., Banday, A. J., Bennett, C. L., et al. 1996, ApJ, 470, 653

Mather, J. C., Fixsen, D. J., Shafer, R. A., Mosier, C., \& Wilkinson, D. T. 1999, ApJ, 512, 511

Olive, K. A., \& Steigman, G. 1995, ApJS, 97, 49

Page, L., Hinshaw, G., Komatsu, E., et al. 2006, ArXiv Astrophysics e-prints Peebles, P. J. E. 1968, ApJ, 153, 1

Peebles, P. J. E., Seager, S., \& Hu, W. 2000, ApJ, 539, L1

Readhead, A. 2007, private communication

Righi, M., Hernandez-Monteagudo, C., \& Sunyaev, R. 2007, ArXiv e-prints, 707

Rubiño-Martín, J. A., Chluba, J., \& Sunyaev, R. A. 2006, MNRAS, 371, 1939

Rubino-Martin, J. A., Chluba, J., \& Sunyaev, R. A. 2007, ArXiv e-prints, 711

Schäfer, B. M., Pfrommer, C., Bartelmann, M., Springel, V., \& Hernquist, L. 2006, MNRAS, 370, 1309

Seager, S., Sasselov, D. D., \& Scott, D. 1999, ApJ, 523, L1

Seager, S., Sasselov, D. D., \& Scott, D. 2000, ApJS, 128, 407

Seljak, U., Sugiyama, N., White, M., \& Zaldarriaga, M. 2003, Phys. Rev. D, 68, 083507

Silk, J. 1968, ApJ, 151, 459

Springel, V., White, M., \& Hernquist, L. 2001, ApJ, 549, 681

Steigman, G. 2006, J. Cosmol. Astro-Part. Phys., 10, 16

Sunyaev, R. A., \& Zeldovich, Y. B. 1970, Ap\&SS, 7, 3

Sunyaev, R. A., \& Zeldovich, Y. B. 1972, Comm. Astrophys. Space Phys., 4, 173

Switzer, E. R., \& Hirata, C. M. 2007a, ArXiv Astrophysics e-prints

Switzer, E. R., \& Hirata, C. M. 2007b, ArXiv Astrophysics e-prints

Wong, W. Y., \& Scott, D. 2007, MNRAS, 375, 1441

Zeldovich, Y. B., Kurt, V. G., \& Syunyaev, R. A. 1968, Zh. Eksperim. Teoretich. Fiziki, 55, 278 